\title{
Unification of Electromagnetism and Gravitation
}

\author{
Raymond J. Beach \\ Lawrence Livermore National Laboratory, L-465, 7000 East Avenue, Livermore, \\ CA 94551 \\ E-mail: beach2@,llnl.gov
}

Using four field equations, a recently proposed theory that covers the phenomenology of classical physics at the level of the Maxwell and Einstein Field Equations (M\&EFEs) but then goes further by unifying electromagnetic and gravitational phenomena in a fundamentally new way is reviewed. Maxwell's field equations are a consequence of the new theory, as are Einstein's field equations augmented by a term that can replicate both dark matter and dark energy. To emphasize the unification brought to electromagnetic and gravitational phenomena by the new theory, specific solutions for a spherically-symmetric charged particle, and both electromagnetic and gravitational waves are presented. A unique feature of the new theory is the treatment of both charge and mass density as dynamic fields, this as opposed to their treatment in the classical M\&EFEs where they are external parameters. This feature enables a procedure for quantizing the mass, charge and angular momentum of particle-like solutions. Finally, antimatter is naturally accommodated by the new theory and its interaction with a gravitational field is reviewed.

(key words: unified field theory, electromagnetism, Maxwell's equations, gravitation, General Relativity, Einstein's field equations, gravitational radiation, hidden variable, dark matter, cosmological constant) 


\section{Introduction}

Assuming the geometry of nature is Riemannian with four dimensions, the following four equations have been proposed as an alternative description of classical physics at the level of the M\&EFEs, ${ }^{i}$ but one that then goes further by reconciling gravity and electromagnetism

$$
\begin{gathered}
F_{\mu v ; \kappa}=a^{\lambda} R_{\lambda k \mu v} \\
a^{\lambda} R_{\lambda}{ }^{v}=\rho_{c} u^{v} \\
u^{\lambda} u_{\lambda}=-1 \\
\left(\rho_{m} u^{\mu} u^{v}+F_{\lambda}^{\mu} F^{v \lambda}-\frac{1}{4} g^{\mu v} F^{\rho \sigma} F_{\rho \sigma}\right)_{; v}=0
\end{gathered}
$$

Both equations (1) and (2) are new, as is the vector field $a^{\lambda}$ that appears in them and serves to couple gravitational to electromagnetic phenomena. Equation (1) couples the Maxwell tensor $F_{\mu \nu}$ to the Riemann-Christoffel (R-C) tensor $R_{\lambda k \mu v}$. Equations (2) couples the Ricci Tensor $R_{\lambda}{ }^{v}$ to the coulombic current density $\rho_{c} u^{v}$. Supplementing these first two equations are equations (3) and (4), both of which are well known. Equation (3) normalizes the four-velocity vector field $u^{\lambda}$ that describes the motion of both the charge density field $\rho_{c}$, and the mass density field $\rho_{m}$, which are assumed to be comoving. Equation (4) describes the conservation of energy and momentum for a specific choice of energy-momentum tensor. Much of the discussion that follows will be focused on describing solutions to these equations and demonstrating that such solutions are consistent with those of the classical M\&EFEs, but then go further by unifying electromagnetic and gravitational phenomena. Taken together, the four field equations are used to axiomatically build up a description of nature in terms of the six dynamic fields described in Table I.

\section{Table I. Dynamic fields}

\begin{tabular}{|c|c|c|}
\hline Field & Description & Number of components \\
\hline$g_{\mu v}$ & Metric tensor & 10 \\
\hline$F_{\mu v}$ & Maxwell tensor & 6 \\
\hline
\end{tabular}




\begin{tabular}{|c|c|c|}
\hline$u^{\lambda}$ & Four-velocity & 4 \\
\hline$a^{\lambda}$ & $\begin{array}{c}\text { Four-vector coupling electromagnetism to } \\
\text { gravitation }\end{array}$ & 4 \\
\hline$\rho_{c}$ & Charge density & 1 \\
\hline$\rho_{m}$ & Mass density & 1 \\
\hline \multicolumn{2}{|c|}{ Total number of independent field components } & 26 \\
\hline
\end{tabular}

An outline of the paper is as follows: Using the four fundamental field equations and the properties of the R-C tensor, Maxwell's equations are derived. The classical field theory based on equations (1) through (4) is then shown to be consistent with the requirements of general covariance after taking full account of dependent or constraining equations. Symmetries of the theory important for the treatment of antimatter and how it responds to a gravitational field are then reviewed. A discussion of the Einstein field equations and how they fit into the framework defined by the fundamental field equations (1) through (4) is then given. Next, a soliton solution to the field equations representing a spherically-symmetric charged particle is reviewed. Emphasized in this particle-like solution are the source terms of the electromagnetic and gravitational fields, $\rho_{c}$ and $\rho_{m}$, respectively, which are themselves dynamic fields in the theory; a development which opens the possibility of quantizing such solutions for charge, mass and angular momentum using a set of self-consistency equations that flow from the analysis. Two radiative solutions representing electromagnetic and gravitational waves are then developed and analyzed with an emphasis on the unification that the theory brings to electromagnetic and gravitational phenomena. A discussion of antimatter and how it is accommodated by the new theory is then given. Finally, to gain insight into the numerical solution of the fundamental field equations (1) through (4), an analysis of the Cauchy initial value problem as it applies to them is given in Appendix II.

In this paper geometric units are used throughout along with a metric tensor having signature $[+,+,+,-]$. Commas before tensor indices are used to denote ordinary derivatives while semicolons before tensor indices denote covariant derivatives. Spatial indices run from 1 to 3 , with 4 the time index. For the definitions of the R-C curvature tensor and the Ricci tensor, the conventions used by Weinberg are followed. ${ }^{\text {ii }}$ 
Maxwell's equations from $F_{\mu v ; \kappa}=a^{\lambda} R_{\lambda \kappa \mu v}$ and $\rho_{c} u^{v}=a^{\lambda} R_{\lambda}^{v}$

Equations (1) and (2) which relate the Maxwell tensor derivatives to the R-C tensor and the charge current density to the Ricci tensor, respectively, are the fundamental relationships from which all of Maxwell's equations flow. The derivation of Maxwell's homogenous equation begins with the algebraic property of the $\mathrm{R}-\mathrm{C}$ tensor

$$
R_{\lambda \kappa \mu v}+R_{\lambda \mu v \kappa}+R_{\lambda v \kappa \mu}=0
$$

Contracting (5) with $a^{\lambda}$ gives,

$$
a^{\lambda} R_{\lambda \kappa \mu v}+a^{\lambda} R_{\lambda \mu v \kappa}+a^{\lambda} R_{\lambda v \kappa \mu}=0
$$

Using (1) to substitute $F_{\mu v ; \kappa}$ for $a^{\lambda} R_{\lambda \kappa \mu v}$ leads to

$$
F_{\mu \nu ; \kappa}+F_{v \kappa ; \mu}+F_{\kappa \mu ; \nu}=0
$$

which is Maxwell's homogenous equation. Maxwell's inhomogeneous equation follows from (1) by contracting its $\mu$ and $\kappa$ indices

$$
F_{; \mu}^{\mu v}=-a^{\lambda} R_{\lambda}^{v}
$$

Making the connection between the RHS of (8) and the coulombic current density $\rho_{c} u^{v} \equiv J^{v}$ using (2) then gives the conventional Maxwell-Einstein version of Maxwell's inhomogeneous equation

$$
F_{; \mu}^{\mu v}=-\rho_{c} u^{v}\left(\equiv-J^{v}\right)
$$

Because $F^{\mu v}$ is antisymmetric, the identity $F_{; \mu ; \nu}^{\mu \nu}=0$ is forced, which in turn forces the coulombic charge to be a conserved quantity

$$
\left(\rho_{c} u^{v}\right)_{; v}=\left(a^{\lambda} R_{\lambda}^{v}\right)_{; v}=0
$$

Using equations (2) and (3), the coulombic charge density can be solved for in terms of $a^{\lambda}, u^{\lambda}$ and the Ricci tensor, 


$$
\rho_{c}=\left\{\begin{array}{c} 
\pm \sqrt{a^{\lambda} R_{\lambda}{ }^{v} a^{\sigma} R_{\sigma v}} \\
o r \\
-a^{\lambda} R_{\lambda}^{v} u_{v}
\end{array} .\right.
$$

In the forgoing development, only equations (1), (2) and (3) are fundamental to the new theory. Maxwell's equations (7) and (9), the conservation of the charge (10), and the solution for the coulombic charge density (11), are all consequences of (1), (2) and (3), and the properties of the R-C curvature tensor. These additional equations all represent constraints; any solution of (1), (2) and (3) must also satisfy (7), (9), (10) and (11).

One of the new pieces of physics in the foregoing development is the introduction of the vector field $a^{\lambda}$, a vector field that has no counterpart in the conventionally accepted development of classical physics but here serves to both couple the Maxwell tensor to the metric tensor through (1), and the charge density to the metric tensor through (2). Much of the analysis and discussion that follows will be focused on the impact of $a^{\lambda}$ and how it drives the development of a classical field theory that encompasses the physics covered by the M\&EFEs, but then goes further by unifying gravitational and electromagnetic phenomena.

\section{A classical field theory that unifies electromagnetism and gravitation}

As shown in the preceding section, equations (1), (2) and (3) combined with the properties of the R-C tensor provide a basis for deriving Maxwell's homogenous and inhomogeneous equations in curved space-time. Taking the source terms of the gravitational and electromagnetic fields, $\rho_{m}$ and $\rho_{c}$, respectively, as dynamic fields to be solved for, a classical field theory of gravitation and electromagnetism that is logically consistent with the requirements of general covariance is possible. For a theory to be logically consistent with the requirements of general covariance, the $\mathrm{N}$ dynamical field components of the theory must be underdetermined by N-4 independent equations, the remaining 4 degrees of freedom representing the freedom in the choice of coordinate system.

Table I lists the six dynamic fields of the theory along with the number of independent components that comprise each field, yielding a total of 26 independent field components. Now consider the 
last of the theory's fundamental equations, equation (4), the energy and momentum conservation equation

$$
\left(\rho_{m} u^{\mu} u^{v}+F_{\lambda}^{\mu} F^{v \lambda}-\frac{1}{4} g^{\mu v} F^{\rho \sigma} F_{\rho \sigma}\right)_{; v}=0
$$

The specific form of the energy-momentum tensor in (4) ensures that $\rho_{m}$ is conserved and that there is a Lorentz force law. These two dependent equations are derived by first contracting (4) with $u_{\mu}$ which leads to the conservation of mass

$$
\left(\rho_{m} u^{v}\right)_{; v}=0
$$

and then combining (4) and (12), which leads to the Lorentz force law

$$
\rho_{m} \frac{D u^{\mu}}{D \tau}=\rho_{c} u_{\lambda}^{\lambda} F_{\lambda}^{\mu}
$$

where $\frac{D u^{\mu}}{D \tau} \equiv u_{; \sigma}^{\mu} u^{\sigma}$. A more complete outline of the derivation of (12) and (13) is given in the Appendix I. Table II collects and summarizes the four fundamental equations of the new theory, along with the number of components of each equation.

Table II. Fundamental equations

\begin{tabular}{|c|c|c|}
\hline Equation & Equation number in text & $\begin{array}{c}\text { Number of } \\
\text { components }\end{array}$ \\
\hline$F_{\mu v ; \kappa}=a^{\lambda} R_{\lambda \kappa \mu v}$ & $(1)$ & 24 \\
\hline$a^{\lambda} R_{\lambda}{ }^{v}=\rho_{c} u^{v}\left(\equiv J^{v}\right)$ & $(2)$ & 4 \\
\hline$u^{\lambda} u_{\lambda}=-1$ & $(3)$ & 4 \\
\hline$\left(\rho_{m} u^{\mu} u^{v}+F^{\mu}{ }_{\lambda} F^{v \lambda}-\frac{1}{4} g^{\mu v} F^{\rho \sigma} F_{\rho \sigma}\right)_{; v}=0$ & $(4)$ & 33 \\
\hline
\end{tabular}


The total number of fundamental field equations listed in Table II is 33 which is greater than the 26 field components listed in Table I that need to be solved for. If all the fundamental equations listed in Table II were independent, then the field components listed in Table I would be overdetermined and the theory would not be compatible with the requirements of general covariance. However, not all the 33 equations listed in Table II are independent. As already noted, dependent constraint equations can be derived from the equations listed in Table II and the properties of the R-C curvature tensor. Table III collects these dependent constraint equations along with a brief description of their derivation.

Table III. Dependent equations

\begin{tabular}{|c|c|c|c|}
\hline Equation & $\begin{array}{c}\text { Equation number } \\
\text { in text }\end{array}$ & Derivation & $\begin{array}{l}\text { Number of } \\
\text { components }\end{array}$ \\
\hline$F_{\mu \nu ; \kappa}+F_{v \kappa ; \mu}+F_{\kappa \mu ; \nu}=0$ & (7) & $\begin{array}{c}\text { (1) and } \\
R_{\lambda \kappa \mu v}+R_{\lambda \mu v \kappa}+R_{\lambda v \kappa \mu}=0\end{array}$ & 4 \\
\hline$\left(\rho_{c} u^{v}\right)_{; v}=\left(a^{\lambda} R_{\lambda}^{v}\right)_{; v}=0$ & (10) & (1) and (2) & 1 \\
\hline$\rho_{c}=\left\{\begin{array}{c} \pm \sqrt{a^{\lambda} R_{\lambda}{ }^{v} a^{\sigma} R_{\sigma v}} \\
o r \\
-a^{\lambda} R_{\lambda}^{v} u_{v}\end{array}\right.$ & (11) & (2) and (3) & 1 \\
\hline$\left(\rho_{m} u^{v}\right)_{; \nu}=0$ & (12) & $(4),(3)$ and (7) & 1 \\
\hline$\rho_{m} \frac{D u^{\mu}}{D \tau}=\rho_{c} u_{\lambda}^{\lambda} F_{\lambda}^{\mu}$ & (13) & (4), (3) and (12) & 4 \\
\hline \multicolumn{3}{|c|}{ Total number of equations } & 11 \\
\hline
\end{tabular}

The 11 dependent constraint equations listed in Table III mean that of 33 fundamental equations listed in Table II, only 33-11=22 are independent. These 22 independent equations satisfy the requirements of general covariance for determining the 26 independent field components of Table I. The remaining four degrees of freedom in the solution representing the four degrees of freedom in choosing a coordinate system. To further elucidate the content of the fundamental field 
equations (1) through (4), an outline of their solution when viewed as a Cauchy initial value problem is presented in Appendix II.

\section{Symmetries of the fundamental field equations}

Before leaving the formal description of the fundamental equations listed in Table II, three important symmetries that these equations exhibit are noted. The first of these symmetries corresponds to charge-conjugation

$$
\left(\begin{array}{c}
u^{\lambda} \\
a^{\lambda} \\
F^{\mu v} \\
g_{\mu v} \\
\rho_{c} \\
\rho_{m}
\end{array}\right) \rightarrow\left(\begin{array}{c}
u^{\lambda} \\
-a^{\lambda} \\
-F^{\mu v} \\
g_{\mu v} \\
-\rho_{c} \\
\rho_{m}
\end{array}\right)
$$

the second symmetry corresponds to a matter to antimatter transformation, as will be discussed and justified later

$$
\left(\begin{array}{c}
u^{\lambda} \\
a^{\lambda} \\
F^{\mu v} \\
g_{\mu v} \\
\rho_{c} \\
\rho_{m}
\end{array}\right) \rightarrow\left(\begin{array}{c}
-u^{\lambda} \\
-a^{\lambda} \\
-F^{\mu v} \\
g_{\mu v} \\
\rho_{c} \\
\rho_{m}
\end{array}\right)
$$

and the third symmetry is the product of the first two

$$
\left(\begin{array}{c}
u^{\lambda} \\
a^{\lambda} \\
F^{\mu v} \\
g_{\mu v} \\
\rho_{c} \\
\rho_{m}
\end{array}\right) \rightarrow\left(\begin{array}{c}
-u^{\lambda} \\
a^{\lambda} \\
F^{\mu v} \\
g_{\mu v} \\
-\rho_{c} \\
\rho_{m}
\end{array}\right)
$$


All three transformations (14) through (16) leave the fundamental equations (1) through (4) unchanged. Including an identity transformation with the symmetries (14) through (16) forms a group, the Klein-4 group with the product of any two of the symmetries (14) through (16) giving the remaining symmetry. Note that among the fundamental fields of the theory, only $g_{\mu \nu}$ and $\rho_{m}$ are unchanged by the symmetry transformations. These symmetry transformations will be useful for defining boundary conditions that lead to quantized mass, charge and angular momentum of particle-like solutions, as well as for the treatment of antimatter which will be discussed later in this manuscript.

\section{How do the Einstein field equations comport with the new theory?}

While evident from the preceding discussion that Maxwell's field equations and the classical physics that flows from them are derivable from the fundamental equations (1) through (4), at this point it is not obvious that the same can be said of Einstein's field equations. The particle-like solution to be analyzed in the following section demonstrates that the Reissner-Nordstrom metric is an exact solution of the fundamental field equations (1) through (4), thus establishing that the new theory and classical General Relativity support the same solutions, at least in the case of spherical symmetry. To go further and determine if Einstein's field equations are in fact derivable from the fundamental equations of the new theory, start by considering equation (4). An immediate consequence of (4) with its vanishing covariant divergence is that a number of trivial tensor equations can be written down relating the covariant divergence of the energy momentum tensor used in (4) to various geometric quantities. The simplest example of such a tensor equation is

$$
G_{; v}^{\mu v}=\alpha T_{; v}^{\mu v}
$$

where $G^{\mu v} \equiv R^{\mu v}-\frac{1}{2} g^{\mu v} R$ is the Einstein tensor, $\alpha$ is an arbitrary constant, and $T^{\mu v}$ is the stress energy tensor defined in (4)

$$
T^{\mu v} \equiv \rho_{m} u^{\mu} u^{v}+F_{\lambda}^{\mu} F^{v \lambda}-\frac{1}{4} g^{\mu v} F^{\rho \sigma} F_{\rho \sigma} .
$$


While (17) is rigorously true, it's trivially satisfied because both sides are independently 0 , the left being 0 by the Bianchi identity and the right by fundamental equation (4). However, an immediate consequence of equation (17) on taking the anti-covariant derivative of both sides is

$$
G^{\mu v}=\alpha T^{\mu v}+\Lambda^{\mu v}
$$

where $\Lambda^{\mu v}$ is a function of integration forced to have vanishing covariant divergence,

$$
\Lambda_{; v}^{\mu v}=0
$$

In the view being put forth here, (19) is satisfied by any solution of fundamental fields that satisfy equation (4). However, (19) by itself contains no useful information. To see this consider that (19) is trivially satisfied for any $G^{\mu v}$ and $T^{\mu v}$ that go with any specific solution to equations (1) through (4) and any value for the constant $\alpha$ by taking $\Lambda^{\mu v}=G^{\mu v}-\alpha T^{\mu v}$. This also demonstrates that the specific choice for the value of $\alpha$ is completely arbitrary and without physical significance; a change in the value of $\alpha$ is absorbed by a change to $\Lambda^{\mu v}$ such that (19) remains satisfied. This discussion should make clear that (19) is of no value when attempting to find a solution to the fundamental fields. To gain a deeper understanding of how (19) fits into the new theory set $\alpha=-1$, again a choice having no physical significance but one that is convenient because equation (19), except for the appearance of $\Lambda^{\mu v}$, reduces to the Einstein field equations in this case, i.e.,

$$
G^{\mu v}=-T^{\mu v}+\Lambda^{\mu v}
$$

The presence of the function $\Lambda^{\mu v}$ in equation (21) means that (21) is not quite identical to the classical Einstein field equations. However, the required presence of $\Lambda^{\mu v}$ is interesting because it can mimic exactly the properties of dark matter, viz., it is conserved with $\Lambda_{; v}^{\mu v}=0$, it is a source of gravitational fields, and it has no interaction signature beyond the gravitational fields it sources. Finally, note that $\Lambda^{\mu v}$ also includes as a special case, the possibility of a cosmological constant, i.e., $\Lambda^{\mu v}=\lambda g^{\mu v}$.

It is important to note that equation (21) with its auxiliary condition (20) is a consequence of fundamental field equation (4) and the properties of the R-C tensor. With questions today regarding the validity of classical General Relativity beyond the confines of our own solar 
system, ${ }^{\text {iii }}$ the most interesting aspect of $\Lambda^{\mu v}$ in (21) is that it can represent both dark matter and dark energy. Today such terms are appended to the energy-momentum tensor of Einstein's field equations in an ad hoc manner to explain both the flattening of rotational-velocity curves observed on galactic scales, and the accelerating expansion of the universe. So in several respects equation (21), which is a consequence of the new theory, is already accepted and being actively used to analyze gravitational physics problems. Finally, it is important to reiterate that equation (21) standalone is not useful for solving for the metric field (the $g_{\mu v}$ 's) because there is no way a priori to fix $\Lambda^{\mu v}$. Ultimately $\Lambda^{\mu v}$ must be found from a solution to the full set of fundamental field equations (1) through (4), i.e., given a specific solution to the fundamental field equations both $G^{\mu v}$ and $T^{\mu v}$ can be calculated enabling the determination of $\Lambda^{\mu v}$ from $\Lambda^{\mu v}=G^{\mu v}+T^{\mu \nu}$.

\section{Particle-like solution: Electric field, gravitational field, and quantization}

Investigated here is an exact solution of the new theory representing a charged, sphericallysymmetric, particle-like soliton. This example is useful because an exact solution to the fundamental field equations (1) through (4) facilitates a clear comparison between the gravitational and electric fields predicted by the new theory and those predicted by the classical M\&EFEs. To proceed I draw on a solution for a spherically-symmetric charged particle that was previously derived in reference [i]. ${ }^{\mathrm{iv}}$ Working in spherical coordinates $(r, \theta, \phi, t)$, it was shown in reference [i] that the following expressions for the dynamic fields given in Table I are an exact solution to the field equations given in Table II 


$$
\begin{aligned}
& g_{\mu \nu}=\left(\begin{array}{cccc}
\frac{1}{1-\frac{2 m}{r}+\frac{q^{2}}{r^{2}}} & 0 & 0 & 0 \\
0 & r^{2} & 0 & 0 \\
0 & 0 & r^{2} \operatorname{Sin}[\theta]^{2} & 0 \\
0 & 0 & 0 & -\left(1-\frac{2 m}{r}+\frac{q^{2}}{r^{2}}\right)
\end{array}\right) \\
& F_{\mu \nu}=\left(\begin{array}{cccc}
0 & B_{\phi} & -B_{\theta} & E_{r} \\
-B_{\phi} & 0 & B_{r} & E_{\theta} \\
B_{\theta} & -B_{r} & 0 & E_{\phi} \\
-E_{r} & -E_{\theta} & -E_{\phi} & 0
\end{array}\right)=s\left(\begin{array}{cccc}
0 & 0 & 0 & \frac{q}{r^{2}}-\frac{q^{3} / m}{r^{3}} \\
0 & 0 & 0 & 0 \\
0 & 0 & 0 & 0 \\
-\frac{q}{r^{2}}+\frac{q^{3} / m}{r^{3}} & 0 & 0 & 0
\end{array}\right) \\
& u^{\lambda}=s\left(0,0,0, \frac{1}{\sqrt{1-\frac{2 m}{r}+\frac{q^{2}}{r^{2}}}}\right) \\
& a^{\lambda}=s\left(0,0,0, \frac{q}{m}\right) \\
& \rho_{c}=\frac{q^{3}}{m} \frac{\sqrt{1-\frac{2 m}{r}+\frac{q^{2}}{r^{2}}}}{r^{4}} \\
& \rho_{m}=\frac{q^{4}}{m^{2}} \frac{\left(1-\frac{2 m}{r}+\frac{q^{2}}{r^{2}}\right)}{r^{4}}
\end{aligned}
$$

where $s= \pm 1$ as will be explained later. Solution (22) is straightforward to verify by direct substitution into the equations of Table II. ${ }^{\mathrm{v}}$ The physical interpretation of this solution is that of a particle having charge $\pm q$ and mass $m$. Of note is the metric tensor which is identical to the Reissner-Nordstrom metric, establishing that the new theory predicts gravitational fields that are in agreement with the Einstein field equations. Furthermore, the electric field is radial and agrees with the coulomb field of the conventional Maxwell equations to leading order in $1 / r$.

Regarding solution (22), several points are worth emphasizing. First, the fundamental equations in Table II which look very different than do the M\&EFEs give the same solutions for the gravitational field, and the electric field to leading order in $1 / r$ as do the M\&EFEs, at least for the case of the spherically-symmetric charged particle investigated here. This lends credence to 
the claim that the new theory's predictions are consistent with those of the M\&EFEs. Second, solution (22) does not satisfy Einstein's field equations, i.e., $G^{\mu v} \neq-T^{\mu v}$. However, (22) does satisfy $G^{\mu v}=-T^{\mu v}+\Lambda^{\mu v}$ with $\Lambda_{; v}^{\mu v}=0$ as outlined earlier. For completeness the values of $G^{\mu \nu}, T^{\mu v}$ and $\Lambda^{\mu v}$ that go with solution (22) are given here

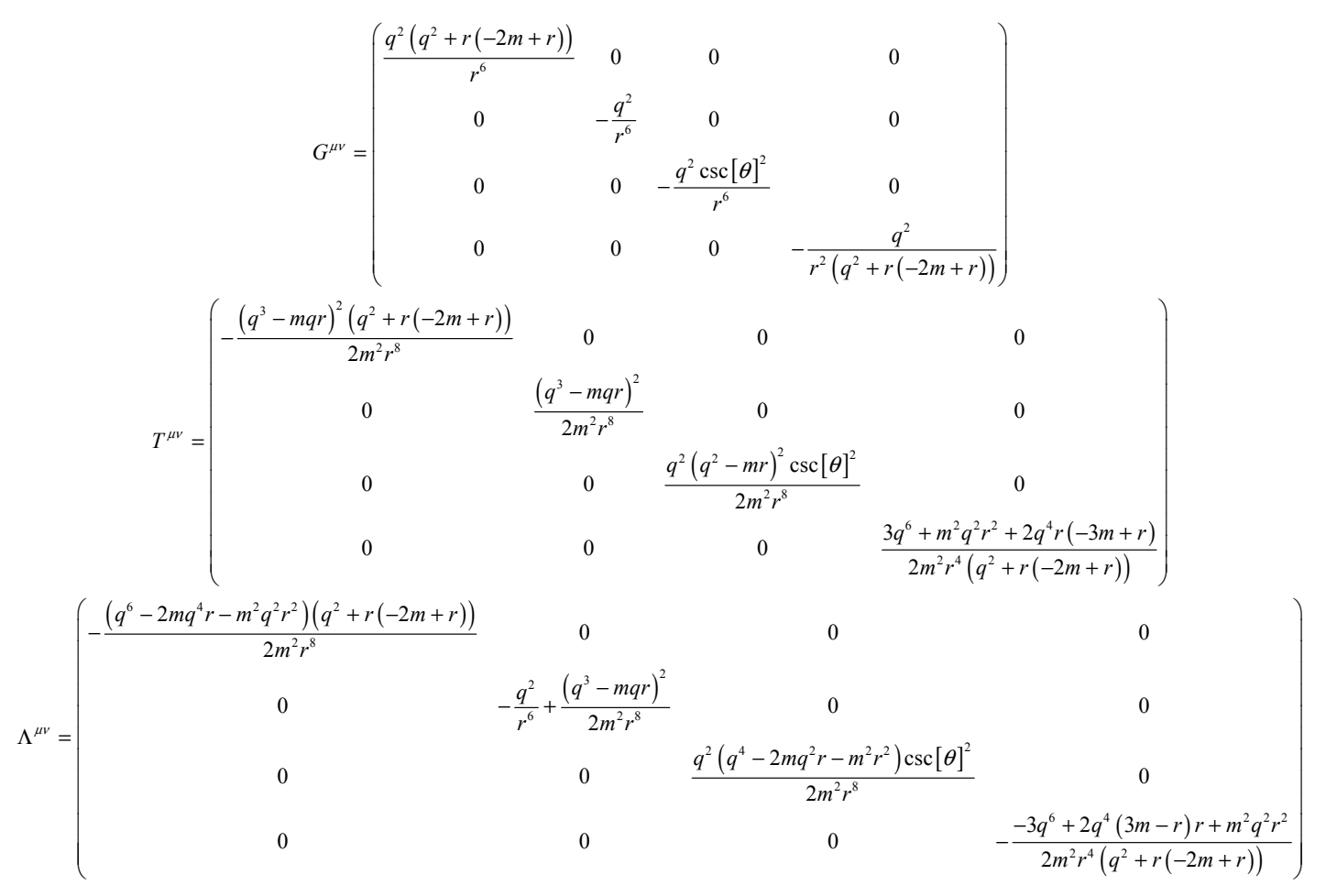

Finally, the new theory's predictions go further than the M\&EFEs by giving the spatial distribution of the mass and charge density as part of their solution, i.e., the mass and charge density are dynamic fields in the new theory. As discussed below, having the mass density and charge density as dynamic fields, when combined with boundary conditions that impose selfconsistency on the field solutions leads to quantization conditions on the particle's mass and charge.

As previously proposed in reference [i], a methodology for quantizing the charge of particle-like solutions such as (22) proceeds by imposing a boundary condition requiring the asymptotic value of the electric field be consistent with the spatially integrated charge density 


$$
q=\int \rho_{c} u^{4} \sqrt{\gamma_{s p}} d^{3} x=\lim _{r \rightarrow \infty} r^{2} F_{14}
$$

where $q$ is the total charge of the particle, $F_{14}$ is the radial electric field component of the Maxwell tensor, and $\gamma_{s p}$ is the determinant of the spatial metric defined by $\mathrm{vi}^{\mathrm{vi}}$

$$
\gamma_{s p i j}=g_{i j}-\frac{g_{i 4} g_{j 4}}{g_{44}}
$$

where $i$ and $j$ run over the spatial dimensions 1,2 and 3. An analogous quantizing boundary condition for the mass of the particle is arrived at by requiring the asymptotic value of its gravitational field be consistent with the spatially integrated mass density of the solution

$$
m=\int \rho_{m}\left|u^{4}\right| \sqrt{\gamma_{s p}} d^{3} x=\lim _{r \rightarrow \infty} r \frac{1+g_{44}}{2}
$$

where $m$ is the total mass of the particle. The reason for the absolute value of $u^{4}$ in the mass boundary condition (26) but not in the charge boundary condition (24) is the symmetry (15) exhibited by the theory's fundamental field equations and the requirement that the boundary conditions exhibit the same symmetry. The boundary conditions (24) and (26) represent selfconsistency constraints on the charge parameter $q$ and the mass parameter $m$ that appear in the metric (22). The proposal here is that these boundary or self-consistency conditions represent additional constraints on physically allowable solutions beyond the fundamental equations presented in Table II.

For the spherically-symmetric solution investigated in (22), the LHS of both (24) and (26) diverge leaving no hope for satisfying those quantization boundary conditions. The upshot of this observation is that while (22) represents a solution that describes the gravitational and electrical fields of a point charge that formally satisfy the equations of the theory in Table II, (22) cannot represent a physically allowed solution. The possibility of finding solutions that satisfy both the equations of the theory in Table II and the quantized charge and mass boundary conditions remains an open question at this point. However, interesting possibilities exist beyond the specific solution investigated here. For example, the modified Reissner-Nordstrom and modified Kerr-Newman metrics developed by S.M. Blinder, ${ }^{\text {vii }}$ give finite values for the LHS of both (24) and (26). Finally, when considering metrics that include nonzero angular momentum, as for example would be 
required for particles having an intrinsic magnetic field, the same methodology used here to quantize the particle's mass and charge can be used to quantize its angular momentum.

The particle-like solution (22) illustrates one restriction that the charge-conjugation symmetry (14) places on metrics that contain a charge parameter $q$. By (14), the charge-conjugation transformation takes $g_{\mu \nu} \rightarrow g_{\mu \nu}$, and $\rho_{c} \rightarrow-\rho_{c}$ or equivalently $q \rightarrow-q$ by (24). This forces the conclusion that the sign of $q$ has no impact on the metric, i.e., the metric can only depend on the absolute value of $q$ since the metric is unchanged by the transformation $q \rightarrow-q$. This result is in line with the known charge containing solutions of the Einstein field equations such as the Reissner-Nordstrom and Kerr-Newman metrics both of which depend on $q^{2}$.

One of the unique features of the classical field theory being proposed here is that it allows for the inclusion of antimatter in a very natural way. The multiplicative factor $s$ in the expressions for $F_{\mu \nu}, a^{\lambda}$ and $u^{\lambda}$ in solution (22) is defined by

$$
s=\left\{\begin{array}{l}
+1 \text { for matter } \\
-1 \text { for antimatter }
\end{array}\right.
$$

and accounts for the matter-antimatter symmetry expressed in (15). The physical interpretation is the $s=-1$ solution represents a particle having the same mass but opposite charge and fourvelocity as the $s=+1$ solution. This is equivalent to the view today that a particle's antiparticle is the particle moving backwards through time. ${ }^{\text {vii }}$ Said another way, the time-like component of the four-velocity is positive for matter and negative for antimatter

$$
u^{4}\left\{\begin{array}{l}
>0 \text { for matter } \\
<0 \text { for antimatter }
\end{array} .\right.
$$

With these definitions for the four-velocity of matter and antimatter, charged mass density can annihilate similarly charged antimass density and satisfy both local conservation of charge (10) and local conservation of mass (12). Additionally, such annihilation reactions conserve total energy by (4).

Because I am endeavoring to develop a theory that flows from the four fundamental equations in Table II axiomatically, an interesting observation is that there appears to be nothing at this point 
in the development that precludes the existence of negative mass density, $\rho_{m}<0$, and negative mass parameter, $m<0$. Indeed, the existence of negative mass in the context of classical General Relativity has been proposed, studied, ${ }^{\mathrm{ix}, \mathrm{x}}$ and invoked when trying to find stable particle-like solutions using the conventional Einstein field equations. ${ }^{\text {xi, xii, xii } H o w e v e r, ~ i n ~ t h e ~ c o n t e x t ~ o f ~ t h e ~}$ present theory, the existence of negative mass density leads to a logical contradiction that can only be resolved by requiring the mass density be non-negative, i.e., $\rho_{m} \geq 0$ always. I'll come back to this point and develop this logical inconsistency when investigating the behavior of matter and antimatter in electric and gravitational fields.

\section{Electromagnetic and gravitational wave solutions}

Working in the weak field limit, derived here are expressions for a propagating electromagnetic plane wave in terms of the vector field $a^{\lambda}$ and the metric tensor $g_{\mu v} \cdot{ }^{\text {xiv }}$ This example is useful as it makes clear the relationship between electromagnetic and gravitational radiation imposed by the fundamental equations in Table II, and predicts that an electromagnetic wave cannot exist without an underlying gravitational wave. To begin, consider an electromagnetic plane wave having frequency $\omega$, propagating in the $+z$-direction and polarized in the $\mathrm{x}$-direction. The Maxwell tensor for this field is given by

$$
F_{\mu \nu}=\left(\begin{array}{cccc}
0 & 0 & -B_{y} & E_{x} \\
0 & 0 & 0 & 0 \\
B_{y} & 0 & 0 & 0 \\
-E_{x} & 0 & 0 & 0
\end{array}\right) e^{i \omega(t-z)}
$$

where $E_{x}$ and $B_{y}$ are the constant field amplitudes. Assuming a near Minkowski weak field metric

$$
\begin{gathered}
g_{\mu v}=\eta_{\mu v}+h_{\mu v} e^{i \omega(t-z)} \\
\left|h_{\mu v}\right| \ll 1
\end{gathered}
$$

where the $h_{\mu v}$ are complex constants, and a constant vector field $a^{\lambda}$,

$$
a^{\lambda}=\left(a^{1}, a^{2}, a^{3}, a^{4}\right)
$$


proceed by substituting for $F_{\mu \nu}, g_{\mu \nu}$ and $a^{\lambda}$ into (1) and only retain terms to first order in the fields $h_{\mu v}$ and $F_{\mu v}$, which are both assumed to be small and of the same order. Doing this leads to a set of 8 independent linear equations for the 16 unknown constants: $h_{\mu v}, a^{\lambda}, E_{x}$ and $B_{y}$. Imposing these constraining equations, then solve for the field components $E_{x}, B_{y}, g_{\mu \nu}$ and $a^{\lambda}$ in terms of 8 free constants

$$
\begin{gathered}
E_{x}=i \omega \frac{\left(h_{11}^{2}+h_{12}^{2}\right)}{2 h_{11}} a^{1} \\
B_{y}=E_{x} \\
g_{\mu \nu}=\eta_{\mu \nu}+\left(\begin{array}{cccc}
h_{11} & h_{12} & -h_{14} & h_{14} \\
h_{12} & -h_{11} & -h_{24} & h_{24} \\
-h_{14} & -h_{24} & h_{33} & -\frac{1}{2}\left(h_{33}+h_{44}\right) \\
h_{14} & h_{24} & -\frac{1}{2}\left(h_{33}+h_{44}\right) & h_{44}
\end{array}\right) e^{i \omega(t-z)},
\end{gathered}
$$

and

$$
a^{\lambda}=\left(a^{1}, a^{1} \frac{h_{12}}{h_{11}}, a^{4}, a^{4}\right) .
$$

This solution illustrates several ways in which the new theory departs from the traditional view of electromagnetic radiation. In the approach being put forth here the undulations in the electromagnetic field are due to undulations in the metrical field (33) via the coupling defined in (1). This result also underlines that the existence of electromagnetic radiation is forbidden in strictly flat space-time. An interesting aspect of this solution is that while electromagnetic radiation necessitates the presence of an underlying gravitational radiation field, the gravitational radiation is not completely defined by the electromagnetic radiation. The supporting gravitational radiation has 6 undetermined constants $\left(h_{11}, h_{12}, h_{14}, h_{24}, h_{33}, h_{44}\right)$, with the only restriction being they satisfy $\left|h_{\mu \nu}\right| \ll 1$ and $h_{11} \neq 0$ as required by (32). Further insight into the content of the metric (33) is evident after making the infinitesimal coordinate transformation from $x^{\mu} \rightarrow x^{\prime \mu}$ given by 


$$
\left(\begin{array}{l}
x \\
y \\
z \\
t
\end{array}\right) \rightarrow\left(\begin{array}{c}
x^{\prime} \\
y^{\prime} \\
z^{\prime} \\
t^{\prime}
\end{array}\right)=\left(\begin{array}{c}
x+\frac{i}{\omega} h_{14} \\
y+\frac{i}{\omega} h_{24} \\
z-\frac{i}{2 \omega} h_{33} \\
t-\frac{i}{2 \omega} h_{44}
\end{array}\right)
$$

and only retaining terms to first order in the $h$ 's. Doing this, the metric (33) is transformed to

$$
g_{\mu \nu}^{\prime}=\eta_{\mu \nu}+\left(\begin{array}{cccc}
h_{11} & h_{12} & 0 & 0 \\
h_{12} & -h_{11} & 0 & 0 \\
0 & 0 & 0 & 0 \\
0 & 0 & 0 & 0
\end{array}\right) e^{i \omega(t-z)}
$$

while $E_{x}^{\prime}$ and $B_{y}^{\prime}$, the transformed electric and magnetic field amplitudes, respectively, are identical to $E_{x}$ and $B_{y}$ given in (32). Note that only the $h_{11}$ and $h_{12}$ components of the metric (36) have an absolute physical significance and $h_{22}=-h_{11}$, which makes the plane wave solution (36) identical to the plane wave solution of the classical Einstein field equations. ${ }^{x v, x v i}$ Because the underlying gravitational wave couples to both charged and uncharged matter, one consequence of the solution here is that there will be an uncertainty when describing the interaction of electromagnetic radiation with matter if the gravitational wave component of the problem is ignored. However, for a nonrelativistic matter, this gravitational interaction (36) vanishes to first order in the $h$ 's. To see this, consider the following expansion of the Lorentz force law

$$
\begin{gathered}
\rho_{m} \frac{D u^{\mu}}{D \tau}=\rho_{c} u_{\lambda}^{\lambda} F_{\lambda}^{\mu} \\
\downarrow \\
\rho_{m} \frac{d u^{\mu}}{d \tau}=-\rho_{m} u^{v} u^{\lambda} \Gamma_{\nu \lambda}^{\mu}+\rho_{c} u^{\lambda} F_{\lambda}^{u}
\end{gathered}
$$

The first term on the RHS in the line above represents the gravitational interaction. This gravitational interaction term vanishes for nonrelativistic matter with $u^{\lambda} \approx(0,0,0,1)$ because for the metric (36) all the $\Gamma_{44}^{\mu}$ vanish to first order in the $h$ 's. 
The forgoing analysis demonstrates the necessity of having an underlying gravitational wave to support the presence of an electromagnetic wave, but the converse is not true, and gravitational radiation can exist independent of any electromagnetic radiation. The following analysis demonstrates this by solving for the structure of gravitational radiation in the absence of electromagnetic radiation. Following the same weak field formalism for the unknown fields $h_{\mu v}$ given in (30), but this time zeroing out $E_{x}$ and $B_{y}$ in (29), leads to the following solutions for $g_{\mu v}$ and $a^{\lambda}$

$$
g_{\mu \nu}=\eta_{\mu \nu}+\left(\begin{array}{cccc}
h_{11} & h_{12} & -h_{14} & h_{14} \\
h_{12} & \frac{h_{12}^{2}}{h_{11}} & -h_{24} & h_{24} \\
-h_{14} & -h_{24} & h_{33} & -\frac{1}{2}\left(h_{33}+h_{44}\right) \\
h_{14} & h_{24} & -\frac{1}{2}\left(h_{33}+h_{44}\right) & h_{44}
\end{array}\right) e^{i \omega(t-z)}
$$

and

$$
a^{\lambda}=\left(a^{1},-a^{1} \frac{h_{11}}{h_{12}}, a^{4}, a^{4}\right)
$$

Both $g_{\mu v}$ given by (38) and $a^{\lambda}$ given by (39) are modified from their solutions in the presence of an electromagnetic wave as given by (33) and (34), respectively. Performing a transformation to the same primed coordinate system as given in (35), here gives the metric field

$$
g_{\mu \nu}^{\prime}=\eta_{\mu \nu}+\left(\begin{array}{cccc}
h_{11} & h_{12} & 0 & 0 \\
h_{12} & \frac{h_{12}^{2}}{h_{11}} & 0 & 0 \\
0 & 0 & 0 & 0 \\
0 & 0 & 0 & 0
\end{array}\right) e^{i \omega(t-z)}
$$

again, illustrating that only the $h_{11}$ and $h_{12}$ components have an absolute physical significance. The interaction of nonrelativistic matter with the gravitational wave (40) vanishes for the same reason that it vanished for the gravitational wave (36) that accompanies electromagnetic radiation. Of 
particular note is the change in the value of the $h_{22}$ component depending on whether the gravitational wave supports an electromagnetic wave as in (36) or is standalone as in (40).

It seems remarkable that the fundamental equations (1) through (4) that lead to Maxwell's equations and electromagnetic radiation can also lead to gravitational waves, unifying both phenomena as undulations of the metric field. On the other hand, equation (1) with $F_{\mu \nu}=0$ is a system of second order partial differential equations, $a^{\lambda} R_{\lambda \kappa \mu v}=0$, in the metric field components $g_{\mu \nu}$ just as the Einstein field equations are, so the fact that both sets of field equations give similar solutions for gravitational waves is not to be completely unexpected. Combining the solutions in this section for gravitational and electromagnetic radiation with those of the last section for the static electric and gravitational fields of a particle-like solution lends additional credence to the claim that the new theory's solutions are consistent with those of the M\&EFEs.

\section{Antimatter and its behavior in electromagnetic and gravitational fields}

The distinction between matter and antimatter is naturally accommodated in the new theory, with antimatter solutions generated from their corresponding matter solutions using transformation (15) - As already mentioned, antimatter can be viewed as matter moving backwards through time. To see this more rigorously, consider the four-velocity associated with a fixed quantity of charge and mass density,

$$
u^{\lambda}=\frac{d x^{\lambda}}{d \tau}
$$

Under the matter-antimatter transformation (15), $u^{\lambda} \rightarrow-u^{\lambda}$, or equivalently $d \tau \rightarrow-d \tau$. This motivates the following expression for the four-velocity in terms of the coordinate time

$$
u^{\lambda}=\frac{d x^{\lambda}}{d \tau}=s \gamma \frac{d x^{\lambda}}{d t}=s \gamma\left(\begin{array}{l}
\boldsymbol{v} \\
1
\end{array}\right)
$$

where $s$ is the matter-antimatter parameter defined in (27), $\boldsymbol{v}=\left(v_{x}, v_{y}, v_{z}\right)$ is the ordinary 3-space velocity of the charge or mass density, and $\gamma=1 / \sqrt{1-v^{2}}$. Equation (42) establishes that the 
corresponding matter-antimatter solutions travel in opposite time directions relative to each other. One of the unusual aspects of the matter-antimatter transformation (15) is that $\rho_{c}$ does not change sign under the transformation. To see that this is consistent with the usual view in which antiparticles have the opposite charge of their corresponding particles, I'll use (42) to illustrate the behavior of a charged matter and antimatter density in an electromagnetic field. Consider a region with an externally defined electromagnetic field

$$
F_{\mu \nu}=\left(\begin{array}{cccc}
0 & B_{z} & -B_{y} & E_{x} \\
-B_{z} & 0 & B_{x} & E_{y} \\
B_{y} & -B_{x} & 0 & E_{z} \\
-E_{x} & -E_{y} & -E_{z} & 0
\end{array}\right)
$$

and with no, or at least a very weak gravitational field so that $g_{\mu \nu} \approx \eta_{\mu \nu}$ and $\Gamma_{\mu \nu}^{\lambda} \approx 0$. Starting with the Lorentz force law (13) and expanding

$$
\begin{gathered}
\rho_{p} \frac{D u^{\mu}}{D \tau}=\rho_{c} u^{\lambda} F_{\lambda}^{\mu} \\
\downarrow \\
\rho_{p} s \gamma \frac{d u_{\mu}}{d t}=\rho_{c} F_{\mu \lambda} u^{\lambda} \\
\downarrow \\
\rho_{p} s \gamma \frac{d}{d t}\left(\begin{array}{c}
s \gamma \vec{v} \\
-s \gamma
\end{array}\right)=\rho_{c}\left(\begin{array}{cccc}
0 & B_{z} & -B_{y} & E_{x} \\
-B_{z} & 0 & B_{x} & E_{y} \\
B_{y} & -B_{x} & 0 & E_{z} \\
-E_{x} & -E_{y} & -E_{z} & 0
\end{array}\right)\left(\begin{array}{l}
s \gamma v_{x} \\
s \gamma v_{y} \\
s \gamma v_{z} \\
s \gamma
\end{array}\right) \\
\downarrow \\
\rho_{p} \frac{d}{d t}\left(\begin{array}{c}
\gamma \vec{v} \\
\gamma
\end{array}\right)=s \rho_{c}\left(\begin{array}{c}
\vec{E}+\vec{v} \times \vec{B} \\
\vec{v} \cdot \vec{E}
\end{array}\right)
\end{gathered}
$$

which on the last line above ends up at the conventional form of the Lorentz force law except for the extra factor of $s$ on the RHS. This factor of $s$ in (44) gives the product $s \rho_{c}$ the appearance that antimatter charge density has the opposite sign to that of matter charge density. The definition of $q$ given in (24) is also equivalent to this point of view because making the matter-antimatter transformation (15) changes the sign of $u^{\lambda}$ but not $\rho_{c}$ in (24), thus changing the sign of $q$. 
Next, I investigate antimatter in a gravitational field. There is no question about the gravitational fields generated by matter and antimatter, they are identical under the matter-antimatter symmetry (15), as $g_{\mu \nu}$ is unchanged by that transformation. To understand whether antimatter is attracted or repelled by a gravitational field, I go again to the Lorentz force law (13), but this time assume there is no electromagnetic field present, just a gravitational field given by a Schwarzschild metric generated by a central mass $m>0$ corresponding to either matter or antimatter. Placing a test particle a distance $r$ from the center of the gravitational field and assuming it to be initially at rest, the equation of motion for the test particle, a geodesic trajectory, is given by the following development

$$
\begin{gathered}
\frac{D u^{\mu}}{D \tau}=0 \\
\downarrow \\
s \gamma \frac{d u^{\mu}}{d t}=-\Gamma^{\mu}{ }_{v \rho} u^{v} u^{\rho} \\
\downarrow \\
s \gamma \frac{d}{d t}\left(s \gamma \frac{d}{d t}\left(\begin{array}{c}
r \\
\theta \\
\phi \\
t
\end{array}\right)\right)=-\Gamma^{\mu}{ }_{v \rho} u^{v} u^{\rho} \approx-\Gamma^{\mu}{ }_{44} s^{2}=\left(\begin{array}{c}
-\frac{m}{r^{2}}\left(1-\frac{2 m}{r}\right) \\
0 \\
0 \\
0
\end{array}\right) s^{2}
\end{gathered}
$$

where in the last line above I have approximated the RHS using the initial at rest value of $u^{\mu}$, $u^{\mu}=(0,0,0, s)$ and additionally used the fact that the only nonzero $\Gamma_{44}^{\mu}$ in a Schwarzschild metric is $\Gamma_{44}^{1}=\frac{m}{r^{2}}\left(1-\frac{2 m}{r}\right)$. Simplifying the LHS of the last line in (45) by noting that initially $\gamma=1$, gives

$$
\frac{d^{2} r}{d t^{2}} \approx-\frac{m}{r^{2}}
$$

independent of $s$, and so demonstrating that the proposed theory predicts both matter and antimatter will be attracted by a gravitational field because they follow the same geodesic trajectory, and this regardless of whether matter or antimatter generated the gravitational field. 
As already noted, there appears to be nothing in the fundamental equations of Table II that preclude the possibility of negative mass density, $\rho_{m}<0$. However, there are inconstancies that are introduced if negative mass density were to exist. As just shown, equation (46) with $m>0$ predicts a test particle at some distance from the origin will feel an attractive gravitational force regardless of whether it is comprised of matter or antimatter. But this attraction is also independent of whether the test particle is comprised of positive or negative mass because the test particle's mass does not enter the calculation; all test particles, regardless of their composition, follow the same geodesic trajectory. Now consider equation (46) with $m<0$. In this case a test particle at some distance from the origin will feel a repulsive gravitational force regardless of whether the test particle is matter or antimatter and regardless of whether the test particle has positive or negative mass. These two situations directly contradict each other, making the new theory logically inconsistent if negative mass density exists. Thus, the only way to avoid this logical contradiction is to require mass density be non-negative always. The condition that $\rho_{m}$ be non-negative always is also consistent with the symmetry transformations (14) through (16) where it was noted that the field $\rho_{m}$ does not change sign under any of the transformations.

\section{Discussion and summary}

Equations (1) and (2) are the most important concepts being proposed in this manuscript, fundamentally tying the Maxwell tensor and charge density to the R-C curvature tensor and thereby unifying electromagnetism with gravitation. The cost of this unification is the introduction of a new field $a^{\lambda}$, a field that has no counterpart in classical physics but in the new theory serves to couple electromagnetic and gravitational phenomena. The unification that ensues puts electromagnetic phenomena on par with gravitational phenomena, with both intrinsically tied to nonzero curvatures. On the surface, this central role for nonzero curvature in all electromagnetic phenomena might be construed as problematic due to the prevalent view today that electromagnetic phenomena can exist in flat space-time. However, looking a little deeper it is not hard to see the connection between the new theory that requires a nonzero curvature for all electromagnetic phenomena and the classical Maxwell theory that works in flat space-time. If one were not aware of the vector field $a^{\lambda}$, but recognized the existence of the coulombic current 
density as is the view today, then that part of the new theory not directly connected to $a^{\lambda}$ is exactly the classical Maxell theory. To see this, consider the equations in Tables II and III that result from replacing $a^{\lambda} R_{\lambda}^{v}$ with the classical coulombic current density, which is just a statement of fundamental equation (2). The before and after equations with this substitution are shown in Tables VI and VII. None of the equations updated with the substitution $a^{\lambda} R_{\lambda}^{v} \rightarrow J^{v}$ reference $a^{\lambda}$ or have an explicit connection to the R-C curvature tensor, and are exactly the classical Maxwell field equations and their consequences.

Table VI. Fundamental equations from Table II with $a^{\lambda} R_{\lambda}{ }^{v} \rightarrow J^{v}$

\begin{tabular}{|c|c|c|}
\hline Original equation & Updated with $a^{\lambda} R_{\lambda}{ }^{v} \rightarrow J^{v}$ & Comment \\
\hline$F_{\mu \nu ; \kappa}=a^{\lambda} R_{\lambda \kappa \mu \nu}$ & $F_{; \mu}^{\mu v}=-J^{v}$ & $\begin{array}{c}\text { Maxwell's } \\
\text { inhomogeneous } \\
\text { equation }\end{array}$ \\
\hline$a^{\lambda} R_{\lambda}{ }^{v}=\rho_{c} u^{v}$ & $J^{v}=\rho_{c} u^{v}$ & $\begin{array}{c}\text { Definition of } \\
\text { classical current } \\
\text { density }\end{array}$ \\
\hline$u^{\lambda} u_{\lambda}=-1$ & $u^{\lambda} u_{\lambda}=-1$ & No change \\
\hline$\left(\rho_{m} u^{\mu} u^{v}+F^{\mu}{ }_{\lambda} F^{\nu \lambda}-\frac{1}{4} g^{\mu \nu} F^{\rho \sigma} F_{\rho \sigma}\right)_{; \nu}=0$ & $\left(\rho_{m} u^{\mu} u^{\nu}+F^{\mu}{ }_{\lambda} F^{\nu \lambda}-\frac{1}{4} g^{\mu v} F^{\rho \sigma} F_{\rho \sigma}\right)_{; \nu}=0$ & No change \\
\hline
\end{tabular}

Table VII. Dependent equations from Table III with $a^{\lambda} R_{\lambda}{ }^{v} \rightarrow J^{v}$

\begin{tabular}{|c|c|c|}
\hline Original equation & Updated with $a^{\lambda} R_{\lambda}{ }^{v} \rightarrow J^{v}$ & Comment \\
\hline$F_{\mu \nu ; \kappa}+F_{\nu \kappa ; \mu}+F_{\kappa \mu ; \nu}=0$ & $F_{\mu \nu ; \kappa}+F_{\nu \kappa ; \mu}+F_{\kappa \mu ; \nu}=0$ & No change \\
\hline$\left(\rho_{c} u^{v}\right)_{; \nu}=\left(a^{\lambda} R_{\lambda}{ }^{v}\right)_{; \nu}=0$ & $J_{; \nu}^{v}=0$ & $\begin{array}{c}\text { Conservation of } \\
\text { charge }\end{array}$ \\
\hline
\end{tabular}




\begin{tabular}{|c|c|c|}
\hline$\rho_{c}=\left\{\begin{array}{c} \pm \sqrt{a^{\lambda} R_{\lambda}{ }^{v} a^{\sigma} R_{\sigma v}} \\
o r \\
-a^{\lambda} R_{\lambda}{ }^{v} u_{v}\end{array}\right.$ & $\begin{array}{c}\text { Definition of } \\
\text { classical current } \\
\text { density }\end{array}$ \\
\hline$\left(\rho_{m} u^{v}\right)_{; \nu}=0$ & $\left(\rho_{m} u^{v}\right)_{; \nu}=0$ & No change \\
\hline$\rho_{m} \frac{D u^{\mu}}{D \tau}=\rho_{c} u^{\lambda} F_{\nu}^{\mu}$ & $\rho_{m} \frac{D u^{\mu}}{D \tau}=\rho_{c} u^{\lambda} F_{\lambda}^{\mu}$ & No change \\
\hline
\end{tabular}

In considering the new theory, one might view classical physics at the level of the Maxwell field equations as they are accepted today as incomplete, there being a hidden field $a^{\lambda}$ that has gone unrecognized. The value of the new theory with its introduction of the vector field $a^{\lambda}$ is the reductionism it brings to classical physics at the level of the M\&EFEs.

In addition to the electromagnetic coverage of the new theory, it also contains solutions replicating those of the Einstein field equations. In fact, the Reissner-Nordstrom metric (and the Schwarzschild metric as a limiting case) are exact solutions of the fundamental equations (1) through (4) demonstrating that the new theory replicates gravitational physics at the level of the Einstein field equations, at least in this spherically-symmetric case. Another reason that the particle-like solution (22) is so important is that it establishes that exact solutions to the theory do exist. This is not at all evident from equation (1), which represents a mixed system of first order partial differential equations for $F_{\mu \nu}$ and so carries with it specific integrability conditions that must be satisfied for solutions to exist. ${ }^{\text {xvii, xviii }}$ The existence of the particle-like solution (22) allays this concern by direct demonstration.

Equation (21), Einstein's field equations with the addition of a function of integration $\Lambda^{\mu v}$ is a consequence of the fundamental field equation (4) and the properties of the R-C tensor. Because the covariant divergence of $\Lambda^{\mu v}$ is constrained to vanish, $\Lambda^{\mu v}$ has built into it all the properties of dark matter and dark energy. Specifically, particular cases of $\Lambda^{\mu v}$ can represent dark matter halos useful for explaining the flattened rotational-velocity curves observed on galactic scales, or the cosmological constant term $\lambda g_{\mu \nu}$ useful for describing dark energy and explaining the accelerating expansion of the universe. The results of applying (21) are all very much in line with current 
investigations of gravitational interactions that use Einstein's field equations with the ad hoc addition of terms to their energy-momentum tensor to account for the effects of dark matter and dark energy. However, in the view of the new theory equation (21) is not required when solving for the fundamental fields listed in Table I. Rather (21) is consequence of the new theory, so any solution of the fundamental field equations (1) through (4) is also a solution of (21) for some choice of $\Lambda^{\mu v}$.

The weak field solution for electromagnetic radiation requires that it be supported by an underlying gravitational radiation. Because of this a test particle in the path of an electromagnetic wave would in addition to feeling the effects of an undulating electromagnetic field, also feel the effects of the underlying gravitational wave. This prediction of the new theory, which does not agree with the classical physics predictions of the M\&EFEs suggests one direction for investigations that could yield empirical results either supporting or refuting the predictions of the new theory. For example, if the new theory is the more correct description of nature, then taking only electromagnetic effects into account for relativistic particles interacting with electromagnetic radiation would introduce an error in the calculated trajectory of particles due to the neglect of the interaction with the underlying gravitational wave.

A detraction of the new theory, at least at its present level of development, is the somewhat ad hoc inclusion of the conserved energy-momentum tensor (4) as one of its four fundamental equations; this rather than a more elegant and integrated variational derivation of the field equations from an appropriate action. The strongest argument for the inclusion of the conserved energy-momentum tensor (4) is the logical completeness it brings to the theory, i.e., the number of independent field equations is four less than the number of independent field variables leaving four degrees of freedom in the determination of the independent field variables; a situation that is consistent with the requirements of general covariance and the freedom in choice of coordinate system. Internal consistency with the requirements of general covariance is a very strong argument for the theory when compared to the situation with the classical M\&EFEs, where, for example, attempts to find particle-like solutions have noted that there is generally one more unknown than there are equations for determining the unknowns. ${ }^{\mathrm{xi}}$

An attractive feature of the new theory is the way in which antimatter is naturally accommodated. Using the matter-antimatter transformation (15), a solution corresponding to antimatter can be 
generated from its symmetric matter solution by changing the sign of $a^{\lambda}, u^{\lambda}$ and $F_{\mu \nu}$, and leaving $\rho_{c}, \rho_{m}$ and $g_{\mu v}$ unchanged. This leads to the same picture as is accepted today in which antimatter density is viewed as matter density moving backwards through time. The change in sign of $u^{\lambda}$ between matter and antimatter ensures that matter density and similarly charged antimatter density can annihilate each other and conserve: charge (10), mass (12), and overall energy (4). By direct calculation, the behavior of matter and antimatter in a gravitational field is demonstrated to be attractive always. Additionally, the mass density field $\rho_{m}$ must be nonnegative always to eliminate the possibility of a logical contradiction within the theory.

The ability of the fundamental field equations (1) through (4) to incorporate a logical description of antimatter is both surprising and unique when one considers that the new theory is a classical field theory and not a quantum field theory. This along with the new theory's introduction of a vector field $a^{\lambda}$ which has no counterpart in the accepted description of classical physics today, and in fact can be considered a hidden variable in that description raises interesting questions regarding how the new theory could potentially be bridged to the quantum mechanical world.

As proposed here, the new theory is a theory of everything at the level of classical physics. This claim rests on the fact that both charge density and mass density are treated as dynamic fields in the theory, leaving no external parameters to be introduced. This of course highlights one shortcoming of the particle-like solution (22). As already noted that solution cannot satisfy the charge and mass boundary conditions (24) and (26), respectively, because the spatial integrals in both of those equations diverge due to singularities at the origin. This is a technical problem due to the metric solution in (22), the Reissner-Nordstrom metric with its singularity at the origin. One way to get around this difficulty might be to investigate other choices of metric such as, for example, the Blinder-Reissner-Nordstrom metric [vii] which is well behaved at the origin. Still other possibilities include relaxing the spherical symmetry of the solutions investigated within to that of cylindrical symmetry, thus allowing for angular momentum about an axis and solutions capable of modeling particles having a magnetic field; but this goes well beyond the level of analysis presented within. 


\section{Conclusion}

The proposed classical field theory of electromagnetism and gravitation developed here encompasses classical physics at the level of the M\&EFEs using the four fundamental field equations as detailed in Table II, but then goes further by unifying electromagnetic and gravitational phenomena in a fundamentally new and mathematically complete way. Maxwell's field equations, and the Einstein field equations with the addition of a term that can mimic dark matter and dark energy are consequences of the four fundamental field equations and the properties of the R-C tensor. The coupling between electromagnetic and gravitational physics is accomplished through the introduction of a vector field $a^{\lambda}$ that has no counterpart in the presently accepted description of nature based on the classical M\&EFEs. In deriving Maxwell's field equations from the new theory it is clear that $a^{\lambda}$ can be viewed as a hidden variable in the classical Maxwell equation description of electromagnetism. This observation explains the apparent contradiction between the new theory's requirement that all electromagnetic phenomena require a nonzero curvature, and the classical Maxwell equation description in which electromagnetic phenomenon can occur is flat space time. Considering gravitational physics in the new theory, both dark matter and dark energy effects are subsumed by the $\Lambda^{\mu v}$ term in (21), a term which is ultimately determined by a solution of the full set of field equations (1) through (4).

The unification brought to electromagnetic and gravitational phenomena by the new theory is demonstrated through several specific examples including the electric and gravitational fields of a spherically-symmetric particle-like solution, and radiative solutions representing both electromagnetic and gravitational waves. One of the strengths of the new theory's field equations, in fact a guiding principle in their development, is the requirement that the full set of fundamental field equations be logically consistent with the requirements of general covariance. Another strength of the new theory is the reductionism brought to electromagnetic and gravitational phenomena by treating the sources of these fields as dynamic variables rather than external parameters that need to be added into the theory; a development which potentially explains the quantization of the mass, charge and angular momentum of particles in the context of a classical field theory. Finally, an outline for numerically solving the fundamental field equations is given in the form of an analysis of the Cauchy initial value problem applied to the fundamental field equations. 
The genesis of the work presented within was reported in a preliminary form in reference [i]. The same fundamental equations and quantizing boundary conditions reviewed here were first reported there. New to this manuscript is the discussion of the symmetries of the fundamental equations in Table II. Based on these symmetry properties, the interpretation of the particle-like solution has been advanced here. The derivation of the Einstein field equations augmented by a function of integration $\Lambda^{\mu v}$ capable of representing dark matter or dark energy is also new to this manuscript. The present manuscript also corrects an error in the weak field analysis of reference [i] leading to the expanded discussion of electromagnetic radiation and its underlying gravitational radiation. Finally, the analysis of the Cauchy initial value problem as it relates to the theory's fundamental equations is new.

\section{Acknowledgement}

For their many useful conversations and critical comments during the preparation of this manuscript I would like to thank my colleagues Charles Boley and Alexander Rubenchik of Lawrence Livermore National Laboratory. This work was performed under the auspices of the U.S. Department of Energy by Lawrence Livermore National Laboratory under Contract DEAC52-07NA27344.

\section{Appendix I - Derivation of the Lorentz force law and the conservation of mass}

The Lorentz force law (13) and the conservation of mass (12) follow from the fundamental equations of the theory. An outline of the derivation of these equations is given here. To derive the conservation of mass equation (12), I begin with equation (4) contracted with $u_{\mu}$

$$
u_{\mu}\left(\rho_{m} u^{\mu} u^{\nu}+F_{\lambda}^{\mu} F^{\nu \lambda}-\frac{1}{4} g^{\mu v} F^{\rho \sigma} F_{\rho \sigma}\right)_{; \nu}=0
$$

Expanding (47) and then simplifying as per the following development 


$$
\begin{aligned}
& u_{\mu}\left(\left(\rho_{m} u^{v}\right)_{; v} u^{\mu}+\rho_{m} u^{v} u_{; v}^{\mu}+F_{\lambda ; v}^{\mu} F^{v \lambda}+F_{\lambda}^{\mu} F_{; v}^{v \lambda}-\frac{1}{2} g^{\mu v} F^{\rho \sigma} F_{\rho \sigma ; v}\right)=0 \\
& \downarrow \\
& \left(\rho_{m} u^{v}\right)_{; \nu}\left(u^{\mu} u_{\mu}\right)+\rho_{m} u^{v}\left(u_{\mu} u_{; \nu}^{\mu}\right)+u_{\mu} F_{\lambda ; \nu}^{\mu} F^{v \lambda}+u_{\mu} F_{\lambda}^{\mu}\left(F_{; v}^{v \lambda}\right)-\frac{1}{2}\left(u_{\mu} g^{\mu \nu}\right) F^{\rho \sigma} F_{\rho \sigma ; v}=0 \\
& \downarrow \\
& \left(\rho_{m} u^{v}\right)_{; v}(-1)+\rho_{m} u^{v}(0)+u_{\mu} F_{\lambda ; v}^{\mu} F^{v \lambda}+u_{\mu} F_{\lambda}^{\mu}\left(-\rho_{c} u^{\lambda}\right)-\frac{1}{2}\left(u^{v}\right) F^{\rho \sigma} F_{\rho \sigma ; v}=0 \\
& \downarrow \\
& -\left(\rho_{m} u^{v}\right)_{; v}+\left(u_{\mu} F_{\lambda ; v}^{\mu} F^{v \lambda}\right)-\rho_{c}\left(u_{\mu} u_{\lambda}^{\lambda} F_{\lambda}^{\mu}\right)-\frac{1}{2}\left(u^{v} F^{\rho \sigma} F_{\rho \sigma ; v}\right)=0 \\
& \downarrow \\
& -\left(\rho_{m} u^{v}\right)_{; v}+u^{\mu} F_{\mu \lambda ; v} F^{v \lambda}-\rho_{c}(0)-\frac{1}{2} u^{\mu} F^{v \lambda} F_{v \lambda ; \mu}=0 \\
& \downarrow \\
& -\left(\rho_{m} u^{v}\right)_{; v}+u^{\mu} F^{v \lambda}\left(F_{\mu \lambda ; v}-\frac{1}{2} F_{v \lambda ; \mu}\right)=0 \\
& \downarrow \\
& -\left(\rho_{m} u^{v}\right)_{; \nu}+u^{\mu} F^{v \lambda}\left(F_{\mu \lambda ; v}+\frac{1}{2} F_{\lambda \mu ; v}+\frac{1}{2} F_{\mu v ; \lambda}\right)=0 \\
& \downarrow \\
& -\left(\rho_{m} u^{v}\right)_{; \nu}+u^{\mu} F^{v \lambda}\left(\frac{1}{2} F_{\mu \lambda ; \nu}+\frac{1}{2} F_{\mu v ; \lambda}\right)=0 \\
& \downarrow \\
& \left(\rho_{m} u^{v}\right)_{; v}=0
\end{aligned}
$$

leads to the conservation of mass equation (12) on the last line above. The Lorentz force law (13) is now derived using the conservation of mass result just derived and equation (4). Expanding and then simplifying per the following development 


$$
\begin{aligned}
& \left(\rho_{m} u^{\mu} u^{v}+F_{\lambda}^{\mu} F^{v \lambda}-\frac{1}{4} g^{\mu v} F^{\rho \sigma} F_{\rho \sigma}\right)_{; v}=0 \\
& \downarrow \\
& \left(\rho_{m} u^{v}\right)_{; \nu} u^{\mu}+\rho_{m} u^{v} u_{; \nu}^{\mu}+F_{\lambda ; \nu}^{\mu} F^{\nu \lambda}+F_{\lambda}^{\mu}\left(F_{; \nu}^{v \lambda}\right)-\frac{1}{2} g^{\mu \nu} F^{\rho \sigma} F_{\rho \sigma ; v}=0 \\
& \downarrow \\
& (0) u^{\mu}+\rho_{m} \frac{D u^{\mu}}{D \tau}+F_{\lambda ; v}^{\mu} F^{v \lambda}-F_{\lambda}^{\mu}\left(\rho_{c} u^{\lambda}\right)-\frac{1}{2} F^{\nu \lambda} F_{v \lambda}^{; \mu}=0 \\
& \downarrow \\
& \rho_{m} \frac{D u^{\mu}}{D \tau}-\rho_{c} u^{\lambda} F_{\lambda}^{\mu}+F^{v \lambda}\left(F_{\lambda ; \nu}^{\mu}-\frac{1}{2} F_{v \lambda}^{; \mu}\right)=0 \\
& \downarrow \\
& \rho_{m} \frac{D u^{\mu}}{D \tau}-\rho_{c} u^{\lambda} F_{\lambda}^{\mu}+F^{\nu \lambda} g^{\mu \sigma}\left(F_{\sigma \lambda ; \nu}-\frac{1}{2} F_{\nu \lambda ; \sigma}\right)=0 \\
& \downarrow \\
& \rho_{m} \frac{D u^{\mu}}{D \tau}-\rho_{c} u^{\lambda} F_{\lambda}^{\mu}+F^{\nu \lambda} g^{\mu \sigma}\left(F_{\sigma \lambda ; v}+\frac{1}{2} F_{\lambda \sigma ; v}+\frac{1}{2} F_{\sigma v ; \lambda}\right)=0 \\
& \downarrow \\
& \rho_{m} \frac{D u^{\mu}}{D \tau}-\rho_{c} u^{\lambda} F_{\lambda}^{\mu}+F^{\nu \lambda} g^{\mu \sigma}\left(\frac{1}{2} F_{\sigma \lambda ; v}+\frac{1}{2} F_{\sigma v ; \lambda}\right)=0 \\
& \downarrow \\
& \rho_{m} \frac{D u^{\mu}}{D \tau}-\rho_{c} u^{\lambda} F_{\lambda}^{\mu}=0
\end{aligned}
$$

leads to the Lorentz force law (13) on the last line above.

\section{Appendix II - The Cauchy problem applied to the fundamental field equations}

One of the unusual features of the field equations in Table II is the lack of any explicit derivatives of the vector field $a^{\lambda}$, a situation which raises questions about the time dependent development of $a^{\lambda}$. To further elucidate this and other questions regarding solutions of the fundamental field equations and to outline how they could be solved numerically, they are here analyzed in terms of a Cauchy initial value problem. 
Given a set of initial conditions comprising the values of the fundamental fields in Table I at all spatial locations, a procedure is outlined that propagates those fields to any other time. To begin, assume $g_{\mu v}, F_{\mu \nu}, u^{\lambda}, \rho_{c}, \rho_{m}$ and $\frac{\partial g_{\mu \nu}}{\partial t}$ are known at all spatial coordinates at some initial coordinate time $t_{0}$. Note that the initial values for the field $a^{\lambda}$ are not required, rather they will be solved for using equation (1) as described below. Also note that in addition to $g_{\mu \nu}$ the initial values of $\frac{\partial g_{\mu v}}{\partial t}$ must be specified because the fundamental field equations are second order in the time derivatives of $g_{\mu \nu}$, a situation analogous to classical General Relativity. The goal of the Cauchy method as it applies here is to start with specified initial conditions for $g_{\mu v}, F_{\mu v}, u^{\lambda}, \rho_{c}, \rho_{m}$ and $\frac{\partial g_{\mu v}}{\partial t}$ at $t_{0}$, and then using the fundamental field equations in Table II solve for $a^{\lambda}, R_{\lambda \kappa \mu v}, \frac{\partial F_{\mu v}}{\partial t}, \frac{\partial u^{\lambda}}{\partial t}, \frac{\partial \rho_{m}}{\partial t}, \frac{\partial \rho_{c}}{\partial t}$ and $\frac{\partial^{2} g_{\mu v}}{\partial t^{2}}$ at $t_{0}$. Armed with these values at $t_{0}$, it is straight forward to propagate the fields $g_{\mu \nu}, F_{\mu \nu}, u^{\lambda}, \rho_{c}, \rho_{m}$ and $\frac{\partial g_{\mu \nu}}{\partial t}$ from their initial conditions at $t_{0}$ to $t_{0}+d t$ and then solve for $a^{\lambda}, R_{\lambda \kappa \mu \nu}, \frac{\partial F_{\mu \nu}}{\partial t}, \frac{\partial u^{\lambda}}{\partial t}, \frac{\partial \rho_{m}}{\partial t}, \frac{\partial \rho_{c}}{\partial t}$ and $\frac{\partial^{2} g_{\mu v}}{\partial t^{2}}$ at $t_{0}+d t$ using the same procedure that was used to find them at $t_{0}$. Repeating this procedure, values for the fundamental fields of the theory can be found at all times. One additional requirement on the field values specified by initial conditions is that they must self-consistent with the fundamental field equations in Table II, i.e., the specified initial conditions must be consistent with a solution to the field equations in Table II.

In what follows Greek indices $(\mu, v, \kappa, \ldots)$ take on the usual spacetime coordinates 1-4 but Latin indices $(i, j, k, \ldots)$ are restricted to spatial coordinates 1-3 only. Since the values of $g_{\mu v}$ and $\frac{\partial g_{\mu v}}{\partial t}$ are known at all spatial coordinates at time $t_{0}$, the values of $\frac{\partial g_{\mu \nu}}{\partial x^{i}}, \frac{\partial^{2} g_{\mu \nu}}{\partial x^{i} \partial x^{j}}$ and $\frac{\partial^{2} g_{\mu v}}{\partial x^{i} \partial t}$ can be calculated at all spatial coordinates at time $t_{0}$. This leaves the ten quantities $\frac{\partial^{2} g_{\mu v}}{\partial t^{2}}$ as the only 
second derivatives of $g_{\mu \nu}$ not known at $t_{0}$. To find the values of $\frac{\partial^{2} g_{\mu v}}{\partial t^{2}}$ at $t_{0}$ proceed as follows. First find the values of the six $\frac{\partial^{2} g_{i j}}{\partial t^{2}}$ at $t_{0}$ using a subset of equations from (1); the subset containing only those equations having spatial derivatives of $F_{\mu \nu}$ on the LHS and at most one time index in each occurrence of the R-C tensor on the RHS. These equations will be used to solve for the values of $a^{\lambda}$ at time $t_{0}$. In all there are 12 such equations out of the 24 that comprise (1) as listed here

$$
\begin{aligned}
& F_{12,1}=a^{\lambda} R_{\lambda 112} \\
& F_{13,1}=a^{\lambda} R_{\lambda 113} \\
& F_{23,1}=a^{\lambda} R_{\lambda 123} \\
& F_{12,2}=a^{\lambda} R_{\lambda 212} \\
& F_{13,2}=a^{\lambda} R_{\lambda 213} \\
& F_{23,2}=a^{\lambda} R_{\lambda 223} \\
& F_{12,3}=a^{\lambda} R_{\lambda 312} \\
& F_{13,3}=a^{\lambda} R_{\lambda 313} \\
& F_{23,3}=a^{\lambda} R_{\lambda 323} \\
& F_{12,4}=F_{24,1}+F_{41,2}=a^{\lambda} R_{\lambda 412} \\
& F_{13,4}=F_{34,1}+F_{41,3}=a^{\lambda} R_{\lambda 413} \\
& F_{23,4}=F_{34,2}+F_{42,3}=a^{\lambda} R_{\lambda 423}
\end{aligned}
$$

The last three equations in (50) use (7), Maxwell's homogenous equation to express the time derivative of a Maxwell tensor component on the LHS as the sum of the spatial derivatives of two Maxwell tensor components. The importance of having only spatial derivatives of the Maxwell tensor components on the LHS of (50) is that they are all known quantities at time $t_{0}$, i.e., since all the $F_{\mu \nu}$ are known at time $t_{0}$, all $\frac{\partial F_{\mu \nu}}{\partial x^{i}}$ can be calculated at time $t_{0}$. Equally important is that the RHS of the 12 equations that comprise (50) contain at most a single time index in each occurrence of their R-C tensor and so are also known at time $t_{0}$. That this is so is seen by examining the general form of the R-C tensor in a locally inertial coordinate system where all first derivatives of $g_{\mu v}$ vanish, i.e., 


$$
R_{\lambda \kappa \mu \nu}=\frac{1}{2}\left(\frac{\partial^{2} g_{\mu \lambda}}{\partial x^{v} \partial x^{\kappa}}-\frac{\partial^{2} g_{\mu \kappa}}{\partial x^{v} \partial x^{\lambda}}-\frac{\partial^{2} g_{\nu \lambda}}{\partial x^{\mu} \partial x^{\kappa}}+\frac{\partial^{2} g_{\kappa \nu}}{\partial x^{\mu} \partial x^{\lambda}}\right)
$$

Note that having at most one time index on the RHS of (51) means that the R-C tensor is made up entirely of terms from $\frac{\partial^{2} g_{\mu v}}{\partial x^{i} \partial x^{j}}$ and $\frac{\partial^{2} g_{\mu v}}{\partial x^{i} \partial t}$, all of which are known at time $t_{0}$. Examining the set of equations (50) there are 12 equations for 4 unknowns, the unknowns being the components of $a^{\lambda}$. These 12 equations can be solved for $a^{\lambda}$ at time $t_{0}$ if the initial conditions were chosen selfconsistently with the fundamental field equations in Table II, i.e., chosen such that a solution to the field equations is indeed possible.

Knowing the $\mathrm{R}-\mathrm{C}$ tensor components with at most one time index at $t_{0}$, I now proceed to determine the $\mathrm{R}-\mathrm{C}$ tensor components with two time indices. Going back to the 24 equations that comprise the set of equations (1), here I collect the subset of those equations in which the LHS is known at time $t_{0}$, i.e., contains only spatial derivatives of the Maxwell tensor, and the RHS has an R-C tensor component that contain two time indices

$$
\begin{aligned}
& F_{14,1}=a^{\lambda} R_{\lambda 114} \\
& F_{24,1}=a^{\lambda} R_{\lambda 124} \\
& F_{34,1}=a^{\lambda} R_{\lambda 134} \\
& F_{14,2}=a^{\lambda} R_{\lambda 214} \\
& F_{24,2}=a^{\lambda} R_{\lambda 224} \\
& F_{34,2}=a^{\lambda} R_{\lambda 234} \\
& F_{14,3}=a^{\lambda} R_{\lambda 314} \\
& F_{24,3}=a^{\lambda} R_{\lambda 324} \\
& F_{34,3}=a^{\lambda} R_{\lambda 334}
\end{aligned}
$$

Each of the equations in (52) contains only one unknown, the R-C component having two time indices. In total, there are six such independent R-C tensor components, 


$$
\begin{aligned}
& R_{1414} \\
& R_{1424} \\
& R_{1434} \\
& R_{2424} \\
& R_{2434} \\
& R_{3434}
\end{aligned}
$$

so the system of nine equations (52) can be algebraically solved for the these six unknown R-C components at time $t_{0}$. With this I now know the value all components of the R-C tensor at time $t_{0}$. From the $t_{0}$ values of the R-C tensor components listed in (53), the values of the six unknown $\frac{\partial^{2} g_{i j}}{\partial t^{2}}$ at $t_{0}$ can be found.

There are three remaining equations from the set of equations (1) that have not yet been addressed

$$
\begin{aligned}
& F_{14,4}=a^{\lambda} R_{\lambda 414} \\
& F_{24,4}=a^{\lambda} R_{\lambda 424} \\
& F_{34,4}=a^{\lambda} R_{\lambda 434}
\end{aligned}
$$

These are the equations for which the temporal derivatives of the Maxwell tensor components are not yet known. Because all values of the R-C tensor and $a^{\lambda}$ are now known at $t_{0}$, these three remaining time-differentiated components of the Maxwell tensor can now be solved for directly using (54), giving complete knowledge $\frac{\partial F_{\mu v}}{\partial t}$ at time $t_{0}$.

If the values of the four $\frac{\partial^{2} g_{\mu 4}}{\partial t^{2}}$ could be calculated then all $\frac{\partial^{2} g_{\mu v}}{\partial t^{2}}$ would be known and all $\frac{\partial g_{\mu v}}{\partial t}$ could be propagated from $t_{0}$ to $t_{0}+d t$. Just as is the case with classical General Relativity, the four $\frac{\partial^{2} g_{\mu 4}}{\partial t^{2}}$ can be determined from the four coordinate conditions that are fixed by the choice of coordinate system. xix Recapping, at $t_{0}$ the following quantities are now known: $g_{\mu v}, F_{\mu \nu}, u^{\lambda}, \rho_{c}, \rho_{m}$ and $\frac{\partial g_{\mu v}}{\partial t}$ are defined by initial conditions, and $a^{\lambda}, \frac{\partial^{2} g_{\mu v}}{\partial x^{\kappa} \partial x^{\lambda}}, R_{\lambda k \mu \nu}$ and $\frac{\partial F_{\mu v}}{\partial x^{\lambda}}$ 
are solved for using those initial conditions, the fundamental field equations and the four coordinate conditions that are fixed by the choice of coordinate system. Still needed to propagate the initial conditions in time from $t_{0}$ to $t_{0}+d t$ are $\frac{\partial u^{\mu}}{\partial t}, \frac{\partial \rho_{m}}{\partial t}$ and $\frac{\partial \rho_{c}}{\partial t}$. Using the Lorentz force law (13), the following progression,

$$
\begin{gathered}
\rho_{m} \frac{D u^{\mu}}{D \tau}=\rho_{c} u^{\lambda} F_{\lambda}^{\mu} \\
\downarrow \\
\rho_{m} u_{; \nu}^{\mu} u^{v}=\rho_{c} u^{\lambda} F_{\lambda}^{\mu} \\
\downarrow \\
\rho_{m} u_{; ;}^{\mu} u^{4}=-\rho_{m} u_{; i}^{\mu} u^{i}+\rho_{c} u^{\lambda} F_{\lambda}^{\mu} \\
\downarrow \\
\rho_{m}\left(\frac{\partial u^{\mu}}{\partial t}+\Gamma_{4 \sigma}^{\mu} u^{\sigma}\right) u^{4}=-\rho_{m} u_{; i}^{\mu} u^{i}+\rho_{c} u^{\lambda} F_{\lambda}^{\mu}
\end{gathered}
$$

shows on the last line above that $\frac{\partial u^{\mu}}{\partial t}$ can be solved for at $t_{0}$ in terms of knowns at $t_{0}$. Using the conservation of mass (12) and knowing $\frac{\partial u^{\mu}}{\partial t}$ at $t_{0}$, the following progression

$$
\begin{gathered}
\left(\rho_{m} u^{v}\right)_{; v}=0 \\
\downarrow \\
\left(\rho_{m} u^{4}\right)_{; 4}=-\left(\rho_{m} u^{i}\right)_{; i} \\
\downarrow \\
\frac{\partial \rho_{m}}{\partial t} u^{4}=-\rho_{m} u_{; 4}^{4}-\left(\rho_{m} u^{i}\right)_{; i}
\end{gathered}
$$

shows on the last line above that $\frac{\partial \rho_{m}}{\partial t}$ can be solved for at $t_{0}$ in terms of knowns at $t_{0}$. Following an analogous progression for $\rho_{c}$ using the charge conservation equation (10), $\frac{\partial \rho_{c}}{\partial t}$ can be solved for at $t_{0}$ in terms of knowns at $t_{0}$. With these, the values of $a^{\lambda}, R_{\lambda \kappa \mu \nu}, \frac{\partial F_{\mu \nu}}{\partial t}, \frac{\partial u^{\lambda}}{\partial t}, \frac{\partial \rho_{m}}{\partial t}, \frac{\partial \rho_{c}}{\partial t}$ and 
$\frac{\partial^{2} g_{\mu v}}{\partial t^{2}}$ are all known at $t_{0}$ and can be used to propagate the initial conditions $g_{\mu \nu}, F_{\mu \nu}, u^{\lambda}, \rho_{c}, \rho_{m}$ and $\frac{\partial g_{\mu v}}{\partial t}$ at $t_{0}$ to time $t_{0}+d t$. Iterating the process then gives the fundamental fields at all times. 


\section{References and endnotes}

\footnotetext{
${ }^{\text {i }}$ Beach, R. (2014) A Classical Field Theory of Gravity and Electromagnetism. Journal of Modern Physics, 5, 928939. doi: $10.4236 /$ jmp.2014.510096.

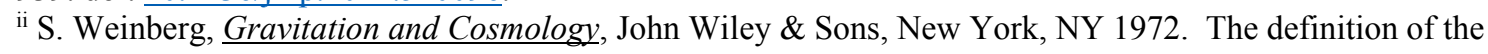

Riemann-Christoffel curvature tensor is $R_{\mu \nu \kappa}^{\lambda} \equiv \partial_{\kappa} \Gamma_{\mu \nu}^{\lambda}-\partial_{\nu} \Gamma_{\mu \kappa}^{\lambda}+\Gamma_{\mu \nu}^{\eta} \Gamma_{\kappa \eta}^{\lambda}-\Gamma_{\mu \kappa}^{\eta} \Gamma_{v \eta}^{\lambda}$, and the definition of the Ricci tensor is $R_{\mu \kappa} \equiv R_{\mu \lambda \kappa}^{\lambda}$.

iii Glenn D. Starkman, “Modifying Gravity: You Can’t Always Get What You Want,” [v1] Mon, 9 Jan 2012 06:46:26 GMT (34kb) arXiv:1201.1697v1 [gr-qc].

iv The interpretation of the solution here is different than in reference [i]. Specifically, in reference [i] the charge density was restricted to be positive, a restriction that is lifted here.

${ }^{v}$ Calculations within were performed in Mathematica: Wolfram Research, Inc., Mathematica, Version 11.0, Champaign, IL (2016).

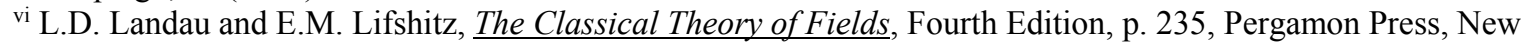
York, NY 1975.

vii Blinder, S. M. "General relativistic models for the electron." Reports on Mathematical Physics 47.2 (2001): 279285.

viii R.P. Feynman, “The Theory of Positrons,” Phys Rev, 1949, 76(6): p. 749-759.

ix Bondi, Hermann. "Negative mass in general relativity." Reviews of Modern Physics 29, no. 3 (1957): 423.

x Bonnor, W. B. "Negative mass in general relativity." General relativity and gravitation 21, no. 11 (1989): 11431157.

${ }^{x i}$ Bailyn, M., and D. Eimerl. "General-Relativistic Interior Metric for a Stable Static Charged Matter Fluid with Large e m." Physical Review D 5, no. 8 (1972): 1897.

xii Bonnor, W.B. and Cooperstock, F.I., 1989. Does the electron contain negative mass? Physics Letters A, 139(9), pp.442-444.

xiii Herrera, L., and V. Verela. "Negative energy density and classical electron models." Physics Letters A 189, no. 12 (1994): 11-14.

xiv This calculation was presented in reference [i] but contained an error that is corrected here. In that original publication, the electric and magnetic fields where not restricted to the same weak field approximation as the $h$ ' $s$. ${ }^{\mathrm{xv}}$ S. Weinberg, Gravitation and Cosmology, John Wiley \& Sons, New York, NY 1972, Section 10.2.

${ }^{x v i}$ L.D. Landau and E.M. Lifshitz, The Classical Theory of Fields, Fourth Edition, p. 235, Pergamon Press, New York, NY 1975, Section 107.

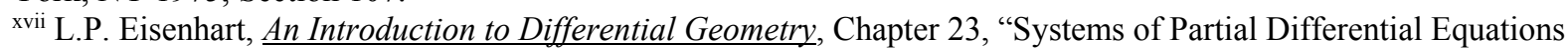
of the First Order, Mixed Systems," Princeton University Press 1947.

xviii For more explanation see the description in reference [i], Section 4. Integrability Conditions.

${ }^{\text {xix }}$ For an excellent discussion of the Cauchy method applied to Einstein's field equations and how, for example, harmonic coordinate conditions determine $\frac{\partial^{2} g_{\mu 4}}{\partial t^{2}}$ see, "The Cauchy Problem," section 5.5 of S. Weinberg, Gravitation and Cosmology, John Wiley \& Sons, New York, NY 1972. 